Supplement of Geosci. Model Dev., 12, 613-628, 2019

https://doi.org/10.5194/gmd-12-613-2019-supplement

(C) Author(s) 2019. This work is distributed under

the Creative Commons Attribution 4.0 License.

(c) (1)

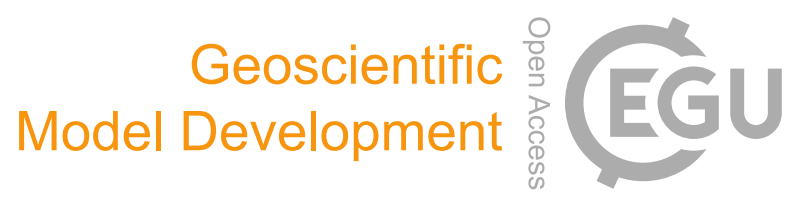

Supplement of

\title{
Topological data analysis and machine learning for recognizing atmospheric river patterns in large climate datasets
}

Grzegorz Muszynski et al.

Correspondence to: Grzegorz Muszynski (gmuszynski@lbl.gov)

The copyright of individual parts of the supplement might differ from the CC BY 4.0 License. 


\section{Supplement Text}

\section{Implementation details}

This supplement describes technical details of a key building blocks that have been used in the implementation of our atmospheric river pattern detection method. This method consists of two stages: feature extraction-topological data analysis

5 (TDA) algorithm and binary classification-support vector machine (SVM) classifier. Below we provide details about the actual implementation-e.g., the data structures and programming languages used, the external software and packages/libraries used for each stage of the method.

\subsection{TDA Algorithm}

The TDA algorithm employs the union-find (often called disjoint-set) data structure to extract topological feature descriptors (connected components) — described in the main manuscript. The data structure and algorithm are implemented in C++ programming language. However, this algorithm uses the very portable language, it is dependent on the custom structures and packages/libraries in The Toolkit for Extreme Climate Analysis (TECA). The TECA is a collection of climate analysis algorithms for extreme event detection and tracking implemented in a scalable parallel framework. More details about the TECA installation can be found in the paper of (Prabhat, et al., 2015) and user manual (https://github.com/LBL-EESA/TECA/blob/

15 master/doc/teca_users_guide.pdf). The code of our algorithm needs to merged with the TECA code and complied all together with it.

\subsection{SVM Classifier}

The binary classification task utilizes SVM classifier (here, based on kernel functions). It employs the C-Support Vector Classifier (C-SVC) from Python scikit-learn that the implementation is based on libsvm. More details about the installation and the use can be found on the sckit-learn and the libsvm websites (https://scikit-learn.org/stable/modules/svm.html, https://www.csie.ntu.edu.tw/ cjlin/libsvm/index.html). 